\title{
Enseñanza del dominio sistema de salud y modelo de atención en las facultades de medicina humana de universidades peruanas, 2020
}

\section{Teaching of the health system and care model domain in the faculties of human medicine of peruvian universities, 2020}

\author{
Palmer J. Hernández-Yépez $z^{1,2, a}$, Sandra S. Chavez-Malpartida, ${ }^{3,4, b}$, Valeria Ayala-Laurel ${ }^{1,2, c}$, \\ Carlos M. Moreno-Arteaga ${ }^{3,4, d}$, Lizbeth V. Zegarra-Quiroz ${ }^{5,6, e}$, Mario J. Valladares-Garrido ${ }^{7, f}$, \\ Fiorella Inga-Berrospi $i^{1,7, g}$ \\ ${ }^{1}$ Facultad de Medicina Humana, Universidad Privada Norbert Wiener. Lima, Perú. \\ ${ }^{2}$ Sociedad Cientifica de Estudiantes de Medicina UNW (SOCIEM-UNW). Lima, Perú. \\ ${ }^{3}$ Facultad de Medicina, Universidad Nacional Mayor de San Marcos. Lima, Perú. \\ ${ }^{4}$ Sociedad Cientifica de San Fernando. Lima, Perú. \\ ${ }^{5}$ Facultad de Medicina, Universidad Católica de Santa María. Arequipa, Perú. \\ ${ }^{6}$ Asociación Cientifico Académica de Estudiantes de Medicina ASOCIADEM. Arequipa, Perú. \\ ${ }^{7}$ Grupo de Investigación en Gestión, Universidad Continental. Lima. Perú. \\ a Estudiante de medicina, ORCID: https://orcid.org/0000-0002-7736-4553 \\ ${ }^{\mathrm{b}}$ Estudiante de medicina, ORCID: https://orcid.org/0000-0003-3519-5189 \\ ${ }^{\circ}$ Estudiante de medicina, ORCID: https://orcid.org/0000-0003-4580-6430 \\ ${ }^{\mathrm{d}}$ Médico cirujano, ORCID: https://orcid.org/0000-0002-8356-1764 \\ ${ }^{e}$ Estudiante de medicina, ORCID: https://orcid.org/0000-0002-2922-7247 \\ ${ }^{\dagger}$ Médico cirujano, maestro en ciencias en investigación epidemiológica. ORCID: https://orcid.org/0000-0003-0839-2419 \\ ${ }^{9}$ Médico cirujano, especialista en gestión en salud. ORCID: https://orcid.org/0000-0002-9778-1557
}

Correspondencia:

Sandra Chavez Malpartida

sandra.chavez@unmsm.edu.pe

Recibido: 25 de julio 2021

Aprobado: 15 de agosto 2021

Publicación en línea: 17 de agosto 2021

Conflictos de interés: Los autores declaran no tener conflictos de interés.

Fuente de financiamiento: Fondo Concursable de Proyectos de Investigación 2019, Universidad Privada Norbert Wiener.

Citar como: Hernández-Yépez $P$, Chavez-Malpartida S, Ayala-Laurel V, Moreno-Arteaga C, ZegarraQuiroz L, Valladares-Garrido M, et al. Enseñanza del dominio sistema de salud y modelo de atención en las facultades de medicina humana de universidades peruanas, 2020. An Fac med. 2021;82(2):171-3.DOI: https://doi. org/10.15381/anales.v82i2.19602

\section{An Fac med. 2021;82(2):171-3. / DOI: https://doi.org/10.15381/anales.v82i2.19602}

Sr. Editor,

En los últimos años han surgido nuevas demandas para los sistemas de salud a nivel mundial, especialmente en países de vías de desarrollo ${ }^{(1)}$. Ante esto, la respuesta ha sido deficiente y se ha identificado a la insuficiente preparación de los profesionales de salud en gestión como un factor importante ${ }^{(2)}$. En el Perú, la obtención de un perfil profesional básico homogéneo del médico egresado es considerado un reto, dado que, la educación médica peruana se enfrenta a una gran variabilidad en la calidad de enseñanza y un enfoque tradicional teórico-técnico basado en contenidos ${ }^{(3,4)}$.

En el 2020, el Ministerio de Salud del Perú (MINSA) aprobó el Documento Técnico “Perfil de competencias esenciales que orienta la formación de los profesionales de la salud. Primera fase: médico y enfermero peruano" (5), que plantea un perfil de aptitudes y dominios que las facultades y escuelas de medicina peruanas deben incluir progresivamente en sus mallas curriculares. Uno de ellos es el dominio de sistema de salud y modelo de atención (SSMA). Los contenidos relacionados a este dominio están orientados a desarrollar la capacidad de liderazgo y permite incluir conceptos como la comunidad y las habilidades interpersonales en la toma de decisiones ${ }^{(1,2)}$. De esta forma, el futuro médico podrá atender adecuadamente las necesidades de cada población. A pesar de la necesidad de incluir estos cursos en la educación médica de pregrado a nivel mundial, su incorporación en las mallas curriculares aún no es homogénea ${ }^{(1,2,6)}$.

En ese sentido, realizamos un estudio que tuvo como objetivo identificar los cursos relacionados al dominio SSMA en las mallas curriculares de las facultades y escuelas de medicina humana de universidades peruanas licenciadas por la Superintendencia Nacional de Educación Superior Universitaria (SUNEDU). Se realizó un estudio observacional, analítico de corte 
transversal. Se recolectó en una base de datos el número total de cursos relacionados al dominio SSMA y las siguientes variables: 1) el tipo de universidad (pública o privada), 2) la ubicación geográfica (Lima o provincia), y 3) el año de creación de cada facultad o escuela de medicina (0 a 25, 26 a 50 o más de 50 años). Asimismo, se evaluó la asociación entre el número de cursos relacionados y las características de las facultades y escuelas mencionadas.

Se seleccionaron las asignaturas que pertenecían al dominio SSMA según lo establecido en el documento técnico anteriormente mencionado ${ }^{(5)}$. Se optó por el consenso de expertos en gestión en salud y educación médica para la selección de los cursos del dominio SSMA en las mallas curriculares, debido al difícil acceso a los sílabos y, por tanto, la imposibilidad de corroborar las competencias establecidas. Estos se relacionaron principalmente con: gerencia en salud, administración en salud, atención primaria en salud, epidemiología y estadística.

De las 143 universidades peruanas licenciadas por SUNEDU, 40 de ellas incluyeron facultades/escuelas de medicina humana. De estas, el 52,5\% pertenecían a universidades públicas; el $70 \%$ se ubicaban fuera de la capital y el $57,5 \%$ no superaban los 25 años de creación. El promedio de cursos del módulo SSMA durante los 7 años de formación médica fue de 11,5; el primer año presentó la mayor media $(2 \pm 1,3)$. En el análisis bivariado, se evidenció una asociación significativa entre el tipo de universidad y la media de cursos del dominio SSMA ( $p=0,042)$. Por el contrario, la ubicación de la universidad $(p=0,234)$ y la antigüedad de cada facultad/escuela de medicina $(p=0.768)$ no mostraron asociación. (Tabla 1).

La distribución geográfica de las facultades y escuelas de medicina en el Perú se ha orientado a responder a las necesidades de cada región ${ }^{(7)}$. Esta tendencia es compatible con nuestros resultados (12 en Lima y 28 en provincia); sin embargo, esta expansión ha propiciado una calidad de educación heterogénea (7). Respecto a los cursos del dominio SSMA, se observó una notoria presencia de es-

Tabla 1. Factores asociados a la frecuencia de cursos en el dominio de sistema de salud y modelo de atención (SSMA) en las facultades de medicina humana de universidades peruanas, 2020

\begin{tabular}{lccc}
\multirow{2}{*}{ Variables } & \multicolumn{2}{c}{ Número de cursos de gestión } & \multirow{2}{*}{$\mathbf{p}^{*}$} \\
\cline { 2 - 3 } & Media & SD** & $0,768^{* * *}$ \\
\hline Antigüedad (categorizada) & & & \\
\hline 0 a 25 años & 11,6 & 11 & \\
\hline 26 a 50 años & 11,7 & 12 & 0,042 \\
\hline Más de 50 años & 10,8 & 10,5 & \\
\hline Tipo & & & 0,234 \\
\hline Pública & 11 & 2,34 & \\
\hline Privada & 12 & 2,48 & \\
\hline Ubicación & & & \\
\hline Provincia & 11 & 2,28 & \\
\hline Lima & 12,5 & 2,96 & \\
\hline
\end{tabular}

* Valores p calculados con la prueba t de Student para varianzas homogéneas.

**SD: desviación estándar.

*** Valor $\mathrm{p}$ calculado con la prueba ANOVA.

tos en las distintas currículas: 1) la cantidad de cursos electivos relacionados varió entre 0 a 7 ; 2) por lo menos un curso relacionado era dictado en cada año lectivo; 3) los cursos relacionados predominaron en los años de formación preclínica. Considerar a estos cursos en los años preclínicos con el objetivo de aplicarlos en los siguientes ha sido enfatizado por centros educativos como la Escuela de Medicina de la Clínica Mayo ${ }^{(8)}$.

Nuestro estudio encontró asociación entre el promedio de asignaturas del dominio SSMA y el tipo de universidad (privada o pública). El contraste en la toma de decisiones y la gestión de los recursos puede explicar este hallazgo. Según la Nueva Ley Universitaria del Perú, todas las universidades tienen autonomía en áreas como lo académico y administrativo ${ }^{(9)}$. Sin embargo, existe dependencia del financiamiento de las universidades públicas en el presupuesto estatal (9). Este escenario podría limitar la implementación de cursos relacionados al dominio SSMA, debido a los costos implicados en el proceso de reforma curricular y la contratación de médicos especialistas, que muchas veces requieren capacitación para adquirir herramientas pedagógicas ${ }^{(10)}$.

Entonces, ¿el médico peruano recién graduado presenta suficientes competen- cias respecto al dominio SSMA?. Probablemente la respuesta no se fundamenta en el número de horas impartidas, sino en calidad de estas. En el año 2005, con el objetivo de analizar la educación médica peruana, se evaluaron las 28 mallas curriculares de las facultades y escuelas de medicina existentes, y las opiniones de docentes expertos y médicos egresados. Se concluyó que "se forman médicos que el Perú ya no necesita", las currículas están sobrecargadas y la enseñanza tiene como metodología central las clases magistrales por parte de médicos especialistas, los cuales han recibido una heterogénea capacitación docente ${ }^{(4)}$.

Este estudio expone la magnitud de la inclusión de cursos relacionados al sistema de salud y modelo de atención en las facultades y escuelas de medicina del país. En base a esto, planteamos como interrogante si la propuesta de una educación médica basada en competencias podría representar el primer paso para el fortalecimiento de nuestro sistema de salud.

\section{AGRADECIMIENTOS}

Al Dr. Guido Bendezú, médico investigador en educación e informática médica, y al Dr. Christian Mejía, médico investigador en sistemas de salud y recursos humanos en salud. 


\section{REFERENCIAS BIBLIOGRÁFICAS}

1. Horton R. A new epoch for health professionals education. Lancet. 2010;376(9756):1875-7. DOI: 10.1016/S0140-6736(10)62008-9

2. Quintero GA. Medical education and the healthcare system - why does the curriculum need to be reformed?. BMC Med. 2014;12(1):1-4. DOI: https:// doi.org/10.1186/s12916-014-0213-3

3. Bermúdez-García A, Allagual de la Quintana A, Farfán-Delgado F. Educación médica en Perú. FEM: Revista de la Fundación Educación Médica. 2020;23(1):5-8. DOI: https://dx.doi.org/10.33588/ fem.231.1041

4. Risco de Domínguez G. Diseño e implementación de un currículo por competencias para la formación de médicos. Rev Peru Med Exp Salud Publica. 2014;31(3):572-81.
5. Ministerio de Salud del Perú [Internet]. Documento Técnico "Perfil de competencias esenciales que orientan la formación de los profesionales de la salud. Primera fase: Médico y Enfermero peruano". Resolución Ministerial No 960-2020. Lima: MINSA; 2020 [Fecha de acceso: 15 de marzo 2021]. Disponible en: https://cdn.www.gob.pe/uploads/document/file/1464482/RM 960-2020-MINSA.PDF.PDF

6. Gonzalo JD, Haidet P, Blatt B, Wolpaw DR. Exploring challenges in implementing a health systems science curriculum: A qualitative analysis of student perceptions. Med Educ. 2016;50(5):523-31. DOI: 10.1111/medu.12957

7. Lip C, Vargas A, Zevallos W, Longa J, Hurtado J Situacion del Profesional Medico Cirujano a Ocho Años de Iniciada la Reforma de la Salud y la Seguridad Social. An la Fac Med. 2000;61(2):99-124 DOI: https://doi.org/10.15381/anales.v61i2.4320
8. Starr S, Agrwal N, Bryan MJ, Buhrman Y, Gilbert J, Huber JM, et al. Science of Health Care Delivery: An Innovation in Undergraduate Medical Education to Meet Society's Needs. Mayo Clin Proc Innov Qual Outcomes. 2017;1(2):117-29. DOI: 10.1016/j. mayocpiqo.2017.07.001

9. Superintendencia Nacional de Educación Superior Universitaria [Internet]. Ley Universitaria $N^{\circ}$ 30220. Lima: Congreso de la Republica; 2014 [Fecha de acceso: 15 de marzo 2021]. Disponible en: https://www.sunedu.gob.pe/wp-content/ uploads/2017/04/Ley-universitaria-30220.pdf

10. Gonzalo JD, Ogrinc G. Health Systems Science: The "broccoli" of Undergraduate Medical Education. Acad Med. 2019;94(10):1425-32. DOI: 10.1097/ACM.0000000000002815 